\title{
Post-extubation Airway Obstruction in Children: Are Steroids the Key to Prevention?
}

\author{
Kaitlin Verdone ${ }^{1}$ and Christopher Watson ${ }^{1}$ \\ ${ }^{1}$ Medical College of Georgia
}

May 2, 2021

\begin{abstract}
Reintubation in the pediatric intensive care unit (PICU) increases morbidity, mortality, and the overall cost of care. Postextubation airway obstruction (PEAO) is a potentially predictable cause of extubation failure and may be prevented by the use of corticosteroids. Defining which patients are most at risk for the development of POAE as well as the optimal dose and timing of corticosteroids for prevention is critical. We review the current literature regarding the use of corticosteroids surrounding extubation in the PICU and discuss the implications that a clear algorithm for identification and treatment of these patients would have in the care of critically ill children.
\end{abstract}

\section{Title:}

Post-extubation Airway Obstruction in Children: Are Steroids the Key to Prevention?

\section{Authors:}

Kaitlin Verdone, DO $^{1}$

Christopher M. Watson, MD, MPH ${ }^{1}$

${ }^{1}$ Department of Pediatrics, Medical College of Georgia at Augusta University, Augusta, Georgia

\section{Correspondence:}

Christopher M. Watson, MD, MPH

Division of Pediatric Critical Care Medicine

Children's Hospital of Georgia

$112015^{\text {th }}$ Street

Augusta, GA 30912

Email: chwatson@augusta.edu

\section{Keywords:}

Dexamethasone, steroid, intubation, post-extubation airway obstruction, post-extubation stridor, children.

\section{Author Contributions:}

Kaitlin Verdone: Conceptualization (equal); writing - original draft (lead); writing review and editing (equal). Christopher M. Watson: Conceptualization (equal); writing - review and editing (equal).

\section{Conflict of Interests:}


The authors declare that there are no conflict of interests.

\begin{abstract}
:
Reintubation in the pediatric intensive care unit (PICU) increases morbidity, mortality, and the overall cost of care. Post-extubation airway obstruction (PEAO) is a potentially predictable cause of extubation failure and may be prevented by the use of corticosteroids. Defining which patients are most at risk for the development of POAE as well as the optimal dose and timing of corticosteroids for prevention is critical. We review the current literature regarding the use of corticosteroids surrounding extubation in the PICU and discuss the implications that a clear algorithm for identification and treatment of these patients would have in the care of critically ill children.

The unanticipated need for reintubation in the pediatric intensive care unit (PICU) is associated with significant increases in morbidity, mortality, and cost of care. ${ }^{1}$ While patients may require reintubation for a multitude of reasons, post-extubation airway obstruction (PEAO) secondary to laryngeal edema is one that may be both predictable and modifiable. ${ }^{2}$ Laryngeal edema can occur from pressure or irritation by an endotracheal tube resulting in stridor or obstruction after extubation. ${ }^{1}$ While this complication occurs in all PICUs, the incidence is highest in low- and middle-income countries due to the presence of unique risk factors. These risk factors include circumstances surrounding intubation itself, such as a higher number of intubations occurring outside of the PICU, as well as patient factors such as malnutrition. ${ }^{2}$ Post-extubation edema is also more commonly symptomatic in children due to differences in airway anatomy including looser submucosal connective tissue that allows fluid to accumulate and a less expandable cricoid cartilage ring with a smaller trachea diameter. In theory, the anti-inflammatory effect of corticosteroids may decrease the amount of local laryngeal inflammation and decrease edema. Several studies have demonstrated that the incidence of PEAO can be decreased by pretreatment with dexamethasone. ${ }^{1}$ However, across these studies, there has been significant dosing variation. Adverse effects associated with high-dose corticosteroid use include hyperglycemia, hypertension, immune suppression and secondary infection, and gastrointestinal bleeding. These risks warrant further investigating what the optimal dose of steroids may be to prevent life-threatening PEAO in children while minimizing the risk of side effects.
\end{abstract}

In this issue of Pediatric Pulmonology, Parajuli et al. evaluate the difference between low-dose (LD; 0.25 $\mathrm{mg} / \mathrm{kg} /$ dose) and high-dose (HD; $0.5 \mathrm{mg} / \mathrm{kg} /$ dose) dexamethasone pretreatment in reducing the risk of PEAO in pediatric patients. Their work follows an initial study from the same institution that showed HD dexamethasone pretreatment reduced the risk and severity of PEAO and need for reintubation. ${ }^{2}$ In this randomized, open-label, non-inferiority trial, the authors report no difference in risk for reintubation due to airway obstruction between the two groups. This is in line with the findings of a recent study in adults that showed no difference between $\mathrm{LD}(5 \mathrm{mg})$ and $\mathrm{HD}(10 \mathrm{mg})$ dexamethasone in preventing PEAO in at risk patients in Taiwan. ${ }^{3}$ Unfortunately, Parajuli et al. were unable to enroll sufficient participants to achieve the desired study power due to self-acknowledged time and logistical constraints. As a result, this study was not adequately powered to prove the non-inferiority of LD versus HD. Of note, in this particular institution the standard is to use dexamethasone pretreatment prior to extubation for every patient intubated for greater than 48 hours. The authors note that this is due to unique factors among their patient population that increase their risk of PEAO, that include a large proportion of patients intubated in an uncontrolled setting outside of an PICU, prolonged periods of time intubated outside of the PICU or receiving hand ventilation, and a large percentage of patients with malnutrition and hypoalbuminemia at time of extubation.

The use of prophylactic corticosteroids prior to extubation is not universal among all PICUs and practice varies broadly with regard not only to dosing regimen but also the identification of at-risk patients. ${ }^{4}$ There are a number of known risk factors for the development of laryngeal edema and post-extubation stridor including upper airway infection, inappropriately large endotracheal tube size or high cuff pressure, traumatic intubation including multiple attempts, prolonged period of intubation, and excessive tube mobility due to improper fixation or patient fighting against the endotracheal tube. Despite these known risk factors, there is no clear system for delineating which patients may benefit from corticosteroid pretreatment prior to extubation. ${ }^{5}$ The air leak test is often employed in an effort to determine which patients are at high risk for 
post-extubation stridor and extubation failure. There is significant variability in how this test is performed and what qualifies as a positive test. Additionally, it has been shown neither to be very sensitive nor specific. ${ }^{6}$ A more promising clinical tool is the novel use of ultrasound to measure laryngeal air column width with the endotracheal cuff inflated versus deflated. When compared with the air leak test in one pediatric study, ultrasound was found to be significantly more sensitive, specific, and accurate in predicting post-extubation stridor. $^{5}$

A Cochrane Systematic Review published in 2009 evaluated the effects of corticosteroids at reducing the risk for stridor and extubation failure in patients of all ages. ${ }^{7}$ No difference in the rates of reintubation was observed when analyzing neonates and children separately. However, they did find a significant reduction in stridor when the neonatal and pediatric patients were analyzed together. The authors of this review concluded that based on the studies available at the time, there was no evidence for the effectiveness of corticosteroids in reducing stridor or extubation failure in neonates and children and therefore there was no consensus for their use in this population. In contrast, a more recently systematic review and meta-analysis of 10 pediatric studies was published in 2020, evaluating the effectiveness of corticosteroids in reducing postextubation stridor and extubation failure in pediatric patients. ${ }^{1}$ Among this larger cohort the investigators noted that, despite a wide range of doses used, corticosteroids did significantly reduce the rates of stridor and reintubation in patients who had - or were at risk of - laryngeal edema. In contrast to the less clear data in pediatrics, there is a larger body of adult data to support the use of corticosteroids for this purpose. A systematic review and meta-analysis of 11 adult trials showed that prophylactic corticosteroids prior to extubation were effective at reducing rates of post-extubation airway obstruction and reintubation in highrisk patients. ${ }^{8}$ These patients were defined as being at-risk based on an air leak test, though the definitions of a positive air leak test varied across the studies.

It is important to note that across all of these studies, in both the pediatric and adult literature, there was no consistency in which corticosteroid was used, dose, frequency, or how much time elapsed between the first dose and timing of extubation. While difficult to draw direct conclusions from these varying regimens regarding optimal dosing, there does seem to be an indication that a single dose of steroids one hour prior to extubation is not effective, but that multiple doses in the 24 hours prior to extubation may have more benefit. ${ }^{3}$ In an effort to answer some of the remaining questions, a multi-center, prospective, double-blind, randomized, placebo-controlled, phase IV clinical trial is now underway in Spain designed to evaluate the effectiveness of steroids versus placebo at preventing upper airway obstruction after extubation in 200 children. ${ }^{9}$ In this study, the investigators plan to use a steroid regimen of dexamethasone at $0.25 \mathrm{mg} / \mathrm{kg} / \mathrm{dose}$ every six hours for a total of four doses, which would fall into line with the LD regimen used in the study by Parajuli et al.

While there currently remain more questions than answers with regard to the use of prophylactic corticosteroids prior to extubation in pediatric patients, a careful review of the literature certainly provides some insight into how to best care for our patients. Based on the current published literature, it appears that the use of corticosteroids prior to a planned extubation is likely beneficial for a select group of at-risk pediatric patients. ${ }^{1}$ While no definitive algorithm for identifying these high risk patients presently exists, in our institution, we combine a careful consideration of any risk factors with an air leak test, in line with what many other institutions presently utilize. ${ }^{10}$ While the choice of corticosteroid is likely of lesser consequence, there seems to be no benefit to support the use of HD over LD steroids. However, it does appear to be important to plan ahead and administer the first dose at least six hours prior to extubation, with more benefit likely occurring with multiple doses given in the 24 hours prior to extubation. ${ }^{3}$ Given the associated increase in healthcare costs and morbidities associated with unanticipated PAEO in children, the present study by Parajuli et al. ultimately draws attention to an important need for continued investigation to better inform the development of evidence-based extubation protocols as well as further mitigation of underlying predisposing factors.

\section{References}

1. Kimura S, Ahn JB, Takahashi M, Kwon S, Papatheodorou S. Effectiveness of corticosteroids for postextubation stridor and extubation failure in pediatric patients: a systematic review and meta-analysis. 
Ann Intensive Care. 2020 Nov 18;10(1):155.

2. Baranwal AK, Meena JP, Singhi SC, Muralidharan J. Dexamethasone pretreatment for $24 \mathrm{~h}$ versus $6 \mathrm{~h}$ for prevention of postextubation airway obstruction in children: a randomized double-blind trial. Intensive Care Med. 2014 Sep;40(9):1285-94.

3. Lin C-Y, Cheng K-H, Kou L-K, Lee C-H. Comparison of High- and Low-dose Dexamethasone for Preventing Post-extubation Airway Obstruction in Adults: A Prospective, Randomized, Double blind, Placebo-controlled Study. Int J Gerontol. 2016 Mar;10(1):11-16.

4. Veldhoen ES, Smulders CA, Kappen TH, Calis JC, van Woensel J, Raymakers-Janssen PA, Bont LJ, Hennus MP. Post-extubation stridor in Respiratory Syncytial Virus bronchiolitis: Is there a role for prophylactic dexamethasone? PLoS One. 2017 Feb 16;12(2):e0172096.

5. El Amrousy D, Elkashlan M, Elshmaa N, Ragab A. Ultrasound-Guided Laryngeal Air Column Width Difference as a New Predictor for Post-extubation Stridor in Children. Crit Care Med. 2018 Jun;46(6):e496-e501.

6. Newth CJ, Hotz JC, Khemani RG. Ventilator Liberation in the Pediatric ICU. Respir Care. 2020;65(10):1601-1610.

7. Khemani RG, Randolph A, Markovitz B. Corticosteroids for the prevention and treatment of postextubation stridor in neonates, children and adults. Cochrane Database Syst Rev. 2009 Jul 8;2009(3):CD001000.

8. Kuriyama A, Umakoshi N, Sun R. Prophylactic Corticosteroids for Prevention of Post-extubation Stridor and Reintubation in Adults: A Systematic Review and Meta-analysis. Chest. 2017 May;151(5):1002-1010.

9. Manrique G, Butragueño-Laiseca L, González R, et al. Effectiveness of steroids versus placebo in preventing upper airway obstruction after extubation in critically ill children: rationale and design of a multicentric, double-blind, randomized study. Trials. 2020 Apr 19;21(1):341.

10. Foland JA, Super DM, Dahdah NS, Mhanna MJ. The use of the air leak test and corticosteroids in intubated children: a survey of pediatric critical care fellowship directors. Respir Care. 2002 Jun;47(6):662-6. 